\title{
All-fiber all-normal dispersion laser with an in-fiber Lyot filter
}

\author{
K. Özgören ${ }^{1}$, F. Ö. Ilday ${ }^{2}$ \\ ${ }^{1}$ Material Science and Nanotechnology Graduate Program, Bilkent University, Ankara, Turkey \\ 2 Physics Department, Bilkent University, Çankaya, Ankara, Turkey \\ *Corresponding author: kozgoren@fen.bilkent.edu.tr
}

\begin{abstract}
We propose use of a short PM-fiber section as birefringent medium to construct an all-fiber Lyot filter, with bandwidth adjustable through the PM-fiber length. An all-fiber all-normaldispersion laser is demonstrated using standard components only.

(C) 2009 Optical Society of America
\end{abstract}

OCIS codes: (140.4050) Lasers, fiber; (140.3510) Mode-locked lasers

\section{Introduction}

In recent years, all-normal-dispersion fiber lasers have attracted much interest because of their simpler cavities compared to other types of fiber lasers [1,2]. The large chirp on the pulse minimizes the nonlinear effects and allows high-energy operation. The mode-locked operation is described well by dissipative soliton formation [3]. In practice, the use of an interference or birefringent filter instead of a grating pair amounts to a certain simplification of the cavity. However, these filters are bulk components, whereas an all-fiber oscillator design is attractive as it would be misalignment free. Various approaches have been pursued to build an all-fiber cavity near $1 \mu \mathrm{m}[4,5,6,7,8]$. However, these required the use of specialty components.

Here, we demonstrate theoretically and experimentally that a short section of polarization-maintaining (PM) fiber spliced to the PM fiber output of an in-fiber polarizing beam splitter (PBS) mimics a Lyot filter [9] with cosine-square filtering profile [10], allowing the construction of an all-fiber all-normal-dispersion Yb-doped fiber laser using standard components. The axes of the two PM fibers are spliced at an angle (nominally $45^{\circ}$ ), thereby coupling light into both axes of the PM-fiber section. Ordinarily this should be followed by a second PBS, however it is not needed for a ring cavity configuration since the first PBS delivers the function of the second polarizer at the end of one round-trip.

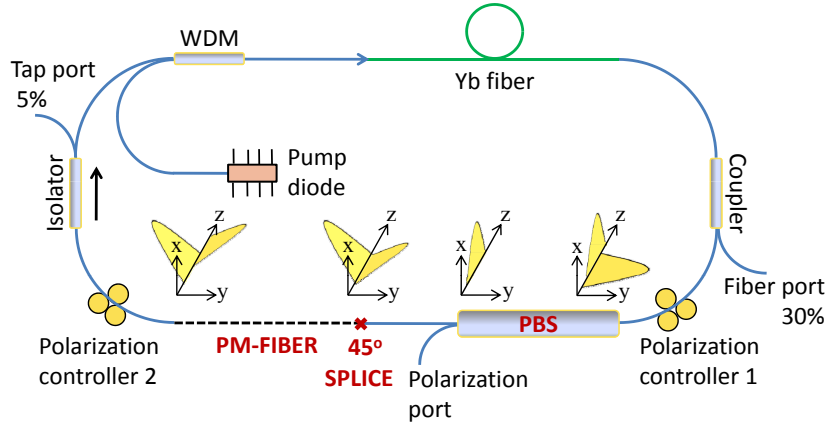

Fig. 1. Sketch of the setup, WDM: wavelength division multiplexer, PBS: polarizing beam splitter. The Lyot filter action is illustrated. Fast axis of the birefringent plate makes $45^{\circ}$ angle with the polarizers.

A schematic of the laser cavity is shown in Fig. 1, with the polarization evolution indicated. The phase difference accumulated between the fast and slow axes over the PM-fiber section is given by $\Delta \phi=(2 \pi / \lambda) L \Delta n$, where $L$ is the length of the PM fiber, $\lambda$ is the wavelength, and $\Delta n=n_{\text {slow }}-n_{\text {fast }}$ is the birefringence. The boundary conditions imposed by the cavity will be the same if the accumulated phase difference $\Delta \phi$ is a multiple of $2 \pi$. Two successive wavelengths satisfying this condition are $\lambda_{m}=L \Delta n / m$ and $\lambda_{m+1}=L \Delta n /(m+1)$ where $m$ is an integer. Assuming $m \gg 1$, the peak-to-peak filtering bandwidth, $\Delta \lambda$, of the PM-fiber section is given by

$$
\Delta \lambda=\lambda_{m}-\lambda_{m+1}=L \Delta n\left(\frac{1}{m}-\frac{1}{m+1}\right) \cong \frac{\lambda^{2}}{L \Delta n}
$$




\section{JTuD54.pdf}
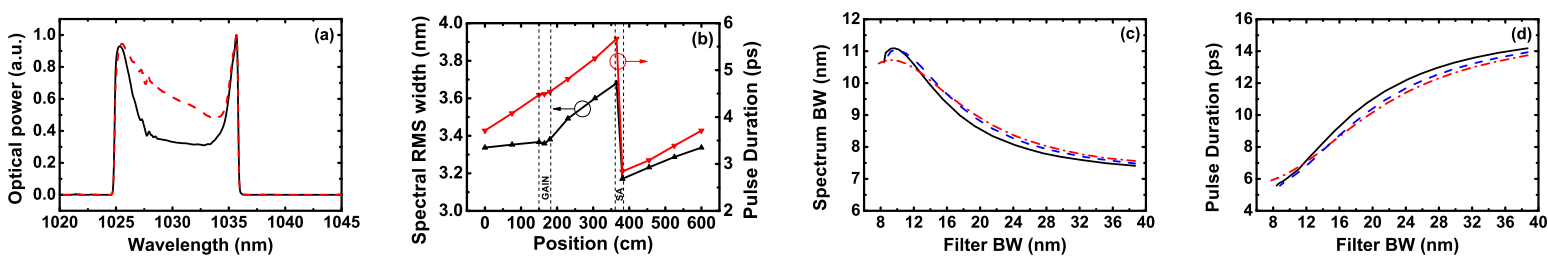

Fig. 2. (a) Simulated laser spectrum after the gain fiber (black line) and after the PM fiber (red line). (b) Simulated spectral bandwidth (black line) and pulse duration (red line) variation over the cavity. Comparison of (c) the spectral width and (d) the pulse duration obtained numerically with the PM-fiber filter (black lines), Gaussian filter (red lines) and cosine-square filter (blue lines).
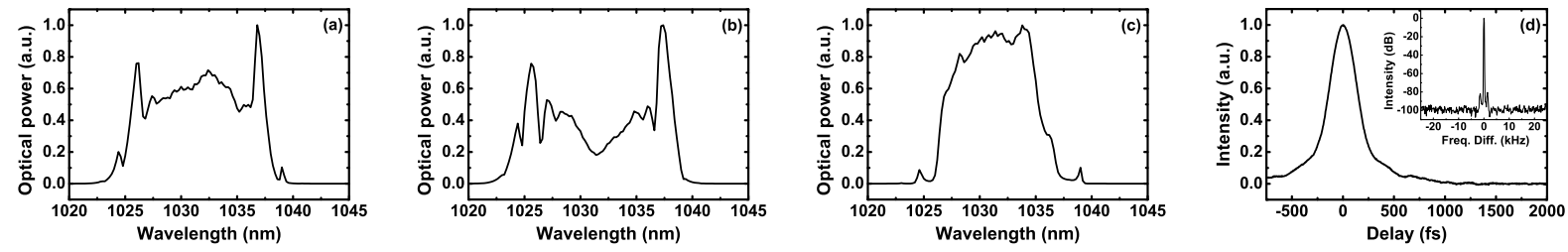

Fig. 3. Measured spectra of pulses for $8.6 \mathrm{~nm}$ effective filter bandwidth from (a) the $30 \%$ fiber port, (b) the polarization extraction port; (c) the $5 \%$ port. (d) Measured autocorrelation of the pulses from the $30 \%$ fiber port. Inset: RF spectrum of the pulses.

Thus, the bandwidth is determined by the length of the PM-fiber section. After the PM fiber, the beam undergoes polarization rotations through the rest of the cavity until it reaches to the PBS again. It can be shown that the peak transmittance wavelength depends on this polarization rotation and the modulation depth depends on the splice angle. We made numerical simulations [11] to check if a stable mode-lock operation is possible against destabilizing effect of the temporal walk-off due to the difference in group velocities along the PM-fiber section. Fig. 2 shows the simulation results obtained with the PM-fiber filter, in comparison to ordinary filters with cosine-square and Gaussian shapes.

Encouraged by the simulation results, we constructed an all-normal-dispersion Yb-fiber laser oscillator (Fig. 1) with a $28 \mathrm{~cm}$ of PM-fiber section, which corresponds to $8.6 \mathrm{~nm}$ filtering bandwidth and $410 \mathrm{fs}$ of temporal walk-off. The laser is mode-locked easily by adjusting the polarization controllers. The output power of the laser is $33 \mathrm{~mW}$ at the $30 \%$ fiber port and $50 \mathrm{~mW}$ at the polarization port at a repetition rate of $34 \mathrm{MHz}$, which corresponds to $1 \mathrm{~nJ}$ pulse energy at the fiber port. The intracavity pulse energy is $3.3 \mathrm{~nJ}$ after the gain fiber. Fig. 4 shows the spectra of the laser obtained at different ports. The laser produces chirped pulses, which are compressed externally using a grating compressor, giving a minimum pulse duration of $230 \mathrm{fs}$, assuming a Gaussian deconvolution factor. The autocorrelator trace of the compressed pulses and high-dynamic range RF spectrum are shown in Fig. 4(d). The RF spectrum of the laser shows $90-\mathrm{dB}$ suppression of the sidebands, which are $1.5 \mathrm{kHz}$ apart. There is no sign of multiple-pulsing at this energy level, which is limited by the maximum available pump power of $300 \mathrm{~mW}$.

In conclusion, we propose that a PM-fiber section inserted into an all-normal-dispersion laser cavity functions as a Lyot filter and stable mode-locked operation is possible, which is confirmed by numerical simulations and experimentally. We also show that the filter bandwidth and modulation depth can be modified by changing the length and splice angle of the PM-fiber section leading to an adjustable filter. We have demonstrated an all-fiber Yb-doped laser cavity using standard components for the first time to our knowledge. The laser generates $1.0 \mathrm{~nJ}$-energy pulses that can be dechirped to $230 \mathrm{fs}$. The pulse energy is limited by available pump power.

\section{References}

1. A. Chong, J. Buckley, W. Renninger, and F. Wise, Opt. Express 14, 10095-10100 (2006).

2. F. W. Wise, A. Chong, and W. H. Renninger, Laser \& Photon. Rev. 2, 58 (2008).

3. W. H. Renninger, A. Chong, and F. W. Wise, Phys. Rev. A 77, 023814 (2008).

4. O. Prochnow, A. Ruehl, M. Schultz, D. Wandt, and D. Kracht, Opt. Express 15, 6889-6893 (2007).

5. C. K. Nielsen and S. R. Keiding, Opt. Lett. 32, 1474-1476 (2007).

6. K. Kieu and F. W. Wise, Opt. Express 16, 11453-11458 (2008).

7. M. Schultz, H. Karow, O. Prochnow, D. Wandt, U. Morgner, and D. Kracht, Opt. Express 16, 19562-19567 (2008).

8. J. Fekete, A. Cserteg and R. Szipocs, Laser Phys. Lett. 1-5 (2008).

9. B. Lyot, C. R. Acad. Sci., 197, pp. 1593-1595 (1933).

10. G. Shabtay, E. Eidinger, Z. Zalevsky, D. Mendlovic and E. Marom, Opt. Express 10, 1534-1541 (2002).

11. P. K. Mukhopadhyay, K. Özgören, I. L. Budunoglu, and F. Ö. Ilday, IEEE J. Sel. Top. Quant. 15, 145 (2009). 